\title{
Stereoscopic filming for investigating evasive side-stepping and anterior cruciate ligament injury risk
}

\author{
Marcus J.C. Lee*a, Paul Bourke ${ }^{\mathrm{b}}$, Jacqueline A Alderson ${ }^{\mathrm{a}}$, David G Lloyd ${ }^{\mathrm{a}}$, Brendan Lay ${ }^{\mathrm{a}}$ \\ ${ }^{a}$ School of Sport Science, Exercise and Health, The University of Western Australia, M408, 35 \\ Stirling Highway, Crawley, Western Australia 6009; \\ ${ }^{\mathrm{b}}$ Western Australian Supercomputer Program, The University of Western Australia, M024, 35 \\ Stirling Highway, Crawley, Western Australia 6009
}

\begin{abstract}
Non-contact anterior cruciate ligament (ACL) injuries are serious and debilitating, often resulting from the performance of evasive sides-stepping (Ssg) by team sport athletes. Previous laboratory based investigations of evasive Ssg have used generic visual stimuli to simulate realistic time and space constraints that athletes experience in the preparation and execution of the manoeuvre. However, the use of unrealistic visual stimuli to impose these constraints may not be accurately identifying the relationship between the perceptual demands and ACL loading during Ssg in actual game environments. We propose that stereoscopically filmed footage featuring sport specific opposing defender/s simulating a tackle on the viewer, when used as visual stimuli, could improve the ecological validity of laboratory based investigations of evasive Ssg. Due to the need for precision and not just the experience of viewing depth in these scenarios, a rigorous filming process built on key geometric considerations and equipment development to enable a separation of $6.5 \mathrm{~cm}$ between two commodity cameras had to be undertaken. Within safety limits, this could be an invaluable tool in enabling more accurate investigations of the associations between evasive Ssg and ACL injury risk.
\end{abstract}

Keywords: Anterior cruciate ligament (ACL) injury, side-stepping (Ssg), stereoscopic applications, realistic visual stimuli, time and space constraints, perceptual skill, stereoscopic filming

\section{INTRODUCTION}

Anterior cruciate ligament (ACL) injuries are arguably one of the most serious, debilitating and costly musculoskeletal injuries that an athlete can suffer from, regardless of the type of sport or level of participation the athlete is involved in. Unfortunately, statistics have shown that a high percentage of athletes who suffer from an ACL injury are relatively young, between the ages of 15 to 25 years old [12]. In addition to the financial burden, many of them also face an increased risk of developing knee joint osteoarthritis in the later stages of their lives [9]. Collectively, the short and long term negative impact that an ACL injury can have on an individual is significant and warrants attention. Hence, there is a need for sport science practitioners to, not only, improve on current diagnostic and treatment measures but to make breakthroughs in developing preventative strategies.

Over the past decade, various research groups have attempted to better understand the aetiology of ACL injuries by investigating the neuromuscular biomechanics related to dynamic movements which load the knee, identifying risk factors and developing injury prevention programs. Unfortunately, despite such efforts, the frequency and severity of ACL injuries have not reduced consistently in team sports such as Australian Rules Football [20], soccer and basketball [1]. From the clinical literature, ACL injuries are typically classified as either contact; i.e. tackle/blow to the knee in soccer; or non-contact; i.e. injury resulting from sudden evasive movements made by the athlete such as side-stepping (Ssg); ,with non-contact injuries making up $50 \%$ to $80 \%$ of all ACL injuries, across a wide domain of sports such as soccer, lacrosse, basketball [19], Australian football [7] and team handball [21]. The high strategy nature of these sports often requires athletes to engage in Ssg manoeuvres during offensive play to evade an oncoming defensive opponent [21] and it is this manoeuvre which has been shown to highly strain the ACL, via a combination of anterior tibial forces, external valgus moments and internal rotation moments about the knee joint [3].

Stereoscopic Displays and Applications XXI, edited by Andrew J. Woods, Nicolas S. Holliman,

Neil A. Dodgson, Proc. of SPIE-IS\&T Electronic Imaging, SPIE Vol. 7524, 752406

(C) 2010 SPIE-IS\&T · CCC code: $0277-786 X / 10 / \$ 18 \cdot$ doi: $10.1117 / 12.840153$ 
During Ssg, the athlete is highly susceptible to an ACL injury due to high external loading applied to the knee and/or inappropriate muscle activation patterns used to stabilise these loads [3] [4] [16]. Despite the suggestion that evasive Ssg entails high ACL injury risk, most players involved in team sports perform the Ssg manoeuvre frequently without getting injured. It is, therefore, important to determine the factors that contribute to the commonly performed Ssg maneouvre becoming injurious. In only two published studies, these critical knee loads have been shown to increase exponentially when there are high temporal [5] and spatial [17] demands that affect the planning of Ssg manoeuvres. Considering that both studies were conducted in a controlled laboratory environment with participants performing Ssg in reaction to generic visual stimuli such as lights, the ever changing temporal and spatial demands posed in a real game environment could make it even more precarious for athletes to side-step safely. Moreover, the use of generic visual stimuli may not be accurately identifying the relationship between the perceptual demands and ACL loading during Ssg in actual game environments. Therefore, realistic visual stimuli, that projects realistic time and space constraints during laboratory testing of athletes performing evasive Ssg, may bring the movement closer to a real life ACL injury scenario. Within safety limits, this could be an invaluable tool in enabling more accurate investigations of the associations between reactive evasive Ssg and ACL injury risk.

Consequently, we chose the sport of soccer, and propose that stereoscopically filmed footage featuring opposing defender/s simulating a tackle on the viewer when presented in the laboratory, could not only present athletes with perceptual conditions similar to those presented in actual game environments, but could potentially elicit Ssg more similar to those performed in real life ACL injury scenarios. Although contextually different, this proposition is supported by previous research suggesting that stereoscopic viewing of converging objects allows enhanced discrimination of time to contact [10]. This is similar to the nature of evasive Ssg tasks whereby athletes usually perform these manouevre in response to converging tackler/tacklers. It is noteworthy that although soccer specific scenarios were chosen as the visual stimuli, the potential for stereoscopically filmed scenarios to be presented as a more realistic form of visual stimuli during laboratory-based investigations of evasive Ssg extends to all other team sports.

A one tackler scenario (1TS) and two tackler scenario (2TS) were filmed. The 1TS featured a defensive player running towards and moving in a defensive manner towards the viewer, Ssg at a predetermined time point in the laboratory, with the viewer's job to avoid the tackler. The 2TS is more complex, featuring two defensive players moving towards the viewer in a defensive manner, from both sides of the viewer's straight line of gaze. At a pre-determined time point, one defensive player veers towards the viewer to simulate a tackle while the other defensive player moves horizontally behind the defensive player making the tackle. In response to the 2TS, the viewers' task is read the tackle as quick as they can and side-step in the opposite direction of the simulated tackle to evade it. The Ss has to be executed within a fixed impact zone where a force plate is located for the collection of foot impact forces, housed within a laboratory which has limited space. Therefore, precise timing of the velocity of approach for both viewer and stimuli, as well as the initiation of the tackle by the stimuli for the elicitation of a reactive side-step by the viewer is crucial, for the successful performance of the Ssg tasks within the impact zone.

The task requirements and the need for accurate stimuli impose a precision not normally encountered in stereoscopic filming for other applications. To present realistic simulations of the tackle scenarios similar to what the viewer would normally encounter in real life, a more rigorous filming process had to be undertaken. The filming process could have been facilitated by purchasing professional cameras that had higher specifications (i.e. better colour responses, higher capture frame rate and genlocking functions). However, these high quality cameras are generally designed to capture content merely for the presentation of depth cues, of which the cues presented, are not necessarily an accurate representation of what is experienced in real life. Moreover, the costs of these cameras are often very high, which potentially limit their applicability and use in research laboratories. Existing commodity cameras were adapted to meet the filming requirements to form a stereoscopic system that will meet the research requirements, which will be discussed in this paper. This includes the development of a customized camera mount which allows two commodity high-definition (HD) cameras to be separated by the average human inter-ocular ocular distance of $6.5 \mathrm{~cm}$, a camera suitability selection process, the calibration and filming procedures involved to achieve an accurate sense of viewing depth and scale, as well as the specific post processing required to arrive at the end product. 


\section{DEVELOPMENT}

\subsection{Key geometric considerations}

In any stereoscopically filmed content, a requirement for achieving an accurate sense of scale and depth is to match the viewing/recording geometry of the cameras to the viewing geometry of the eventual observer. The view frustum is defined as the rectangular cone whose edges are defined by the four rays emanating from each eye to the corners of the viewing screen (Figure 1). This frustum defines what portions of the scene are visible to each eye of the viewer and it is this frustum that needs to be captured with the cameras.

The Ssg tasks require both the viewer and simulated tackler/s to converge simultaneously at similar velocities before a tackle is initiated. The viewer has to read the tackle as quickly as possible and perform an evasive side-step within a fixed impact zone where the force plate is positioned, in a laboratory with limited space. This poses certain challenges not normally encountered when filming standard stereoscopic content whereby the accuracy of depth cues are not a priority. To ensure that the depth information is accurate when the viewer is within the impact zone, a more rigorous filming process involving a substantial amount of equipment development had to be undertaken. The challenges encountered and accompanying solutions will be presented in the following sections.

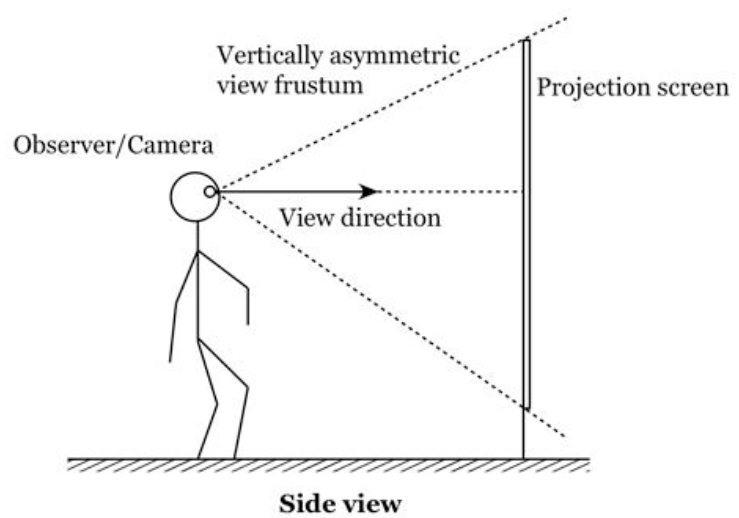

Side view

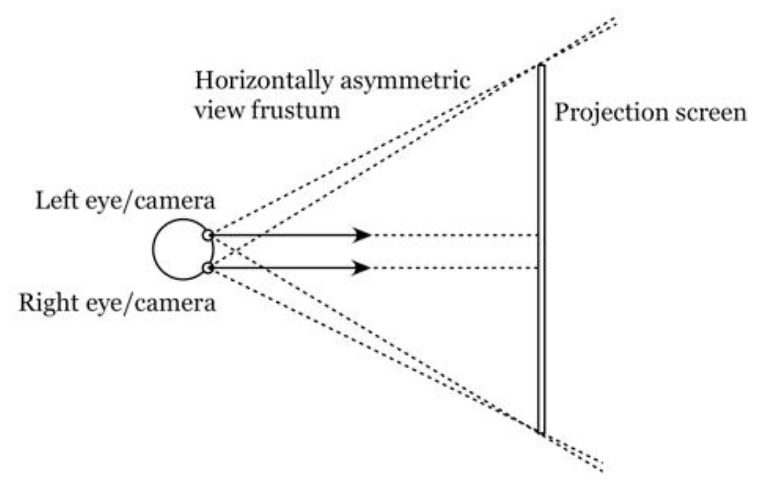

Top view

Figure 1. For a true sense of scale and depth perception the geometry of the observer and intended display surface needs to match the optics of the video camera and projection plane (CCD). The vertical and horizontal asymmetric view frustums are shown on the left and right respectively.

\subsection{Customized dual mount rig}

Stereoscopic filming requires mounting two high definition (HD) cameras on a tripod and filming simultaneously. The visual experience of depth perception when viewing general stereoscopic content can be created using a range of camera separation distances other than eye separation. However, for the creation of the tackle scenarios which possess a sense of three-dimensional (3D) scale and depth similar to what an athlete would see in real life, the centers of both camera lenses need to be representative of the left and right eyes of an average person, which has an inter-ocular separation of approximately $6.5 \mathrm{~cm}$ [23]. This eye separation results in different images being projected to each eye when viewing the same visual field, which is then processed by the visual cortex as one, providing a sense of depth during normal vision [15]. Achieving such a camera separation using a parallel mount configuration poses certain problems, as the physical dimensions of professional cameras generally preclude such a separation when placed side-by-side. This includes the cameras used in the current project. Various solutions exist to overcome this limitation, which include the use of custom offset lenses or a beamsplitter to fold the light path for one of the cameras. However, these solutions involve significant costs or impose other limitations on the filming process. Instead, a more cost effective solution was adopted via the development of a customized dual mount rig.

The dual mount rig enables such a camera separation due to its two plates on which each camera was mounted level. The plates slide along a track such that the cameras can be moved and replaced without destroying alignment. When the 
cameras have been positioned appropriately, the plates can be locked down on the base of the track. However, with both cameras upright, the closest separation attainable still exceeds $8.0 \mathrm{~cm}$.

Due to the asymmetric width on either side of a camera with respect to the center of the lens, a narrower separation could be achieved if one of the cameras was mounted upside down. Therefore, an additional metal arm was built to mount the left camera upside down, before mounting the arm on the plate itself. This reduced the inter-camera separation to a minimum of $6.2 \mathrm{~cm}$, easily achieving $6.5 \mathrm{~cm}$. The dual mount rig with the cameras is then mounted on a tripod for leveling and filming (Figure 2).

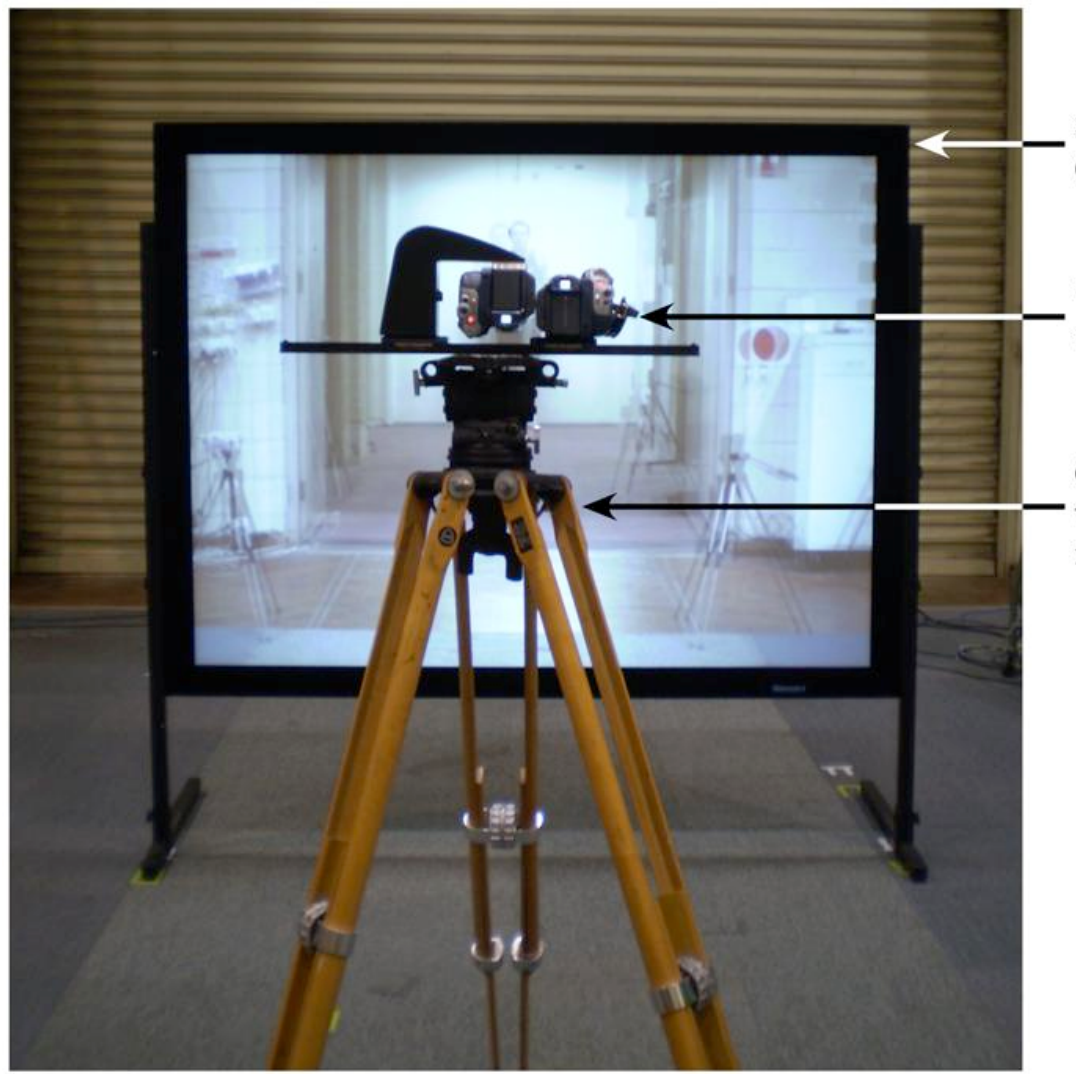

Stereoscopic projection surface.

(Polaroid based, rear projection)

Twin HD camera pair, one inverted to achieve human eye separation.

Camera located at position at which a correct sense of depth is achieved, above the force plate.

Figure 2. The final camera mount (foreground) with the left eye camera inverted so as to achieve average human eye separation. The background shows the screen surface on which the stimuli movies are displayed.

\subsection{Camera pair selection and calibration prior to filming}

The two video cameras used here have a capture rate of 25 frames progressive (25P) and are HD resolution, with the highest available resolution of 1920 pixels x 1080 pixels, a 16:9 aspect ratio. The progressive nature of these cameras allow full frames to be captured instantaneously instead of interlaced fields, which makes them more suitable for recording human motion. Despite using two cameras of the same brand and model, manufacturing inconsistencies resulted in the cameras having their sensors positioned differently with respect to the lens. These differences result in a vertical offset in the captured images of otherwise perfectly parallel and level cameras. The lenses on the cameras also exhibit barrel distortions most evident towards the corners of the images. This distortion does not affect the required stereoscopic content for this project as the information of interest (the simulated tackler/s) are positioned more towards the middle of the image frames and the content on the edges are cropped. It is noteworthy that barrel distortions could equally be corrected during post-processing.

To facilitate calibration, the live stream of the alignment pattern captured from the camera that was mounted upside down was fed into custom software, which automatically rotated the captured image by 180 degrees. Manual adjustments 
were made to the metal arm which held the camera that was upside down till the captured grid images were closely matched between both cameras. The filming and matching of the alignment pattern was performed with three cameras in an attempt to find the camera pair which had the closest matching optics.

Prior to locking down the aligned cameras which operate independently, the cameras' settings were set identically to ensure that a matching image was being captured. An important capture setting that requires matching for comfortable stereo viewing is image colour and brightness. Differences in colour and brightness are significantly more time consuming and problematic to fix in post processing. Therefore, prior to filming, a manual white balancing was performed for both cameras to match them for colour and brightness. The cameras were then set to film at a 16:9 aspect ratio even though the movie playback system is of a 4:3 aspect ratio. This method of filming is advantageous, providing plenty of room for horizontal sliding of the left and right images for aligning zero parallax.

\subsection{Camera setup, scene calibration and filming procedures}

In order to match the camera optics and the eventual viewer geometry, an alignment procedure was conducted prior to filming. The cameras need to be parallel when placed alongside each other, at a height similar to the position of the eventual viewers' eyes from the ground. In this project, an average height of $1.70 \mathrm{~m}$ was chosen to represent the viewing position of an average person's eyes. Regardless of placement height, the cameras have to be perpendicular to the eventual viewing screen when filming content in the frontal plane. This is necessary because the recording plane needs to match the eventual viewing projection plane. Any tilting or rotation of the cameras results in a discrepancy between the recording plane and the eventual projection plane (Figure 3).

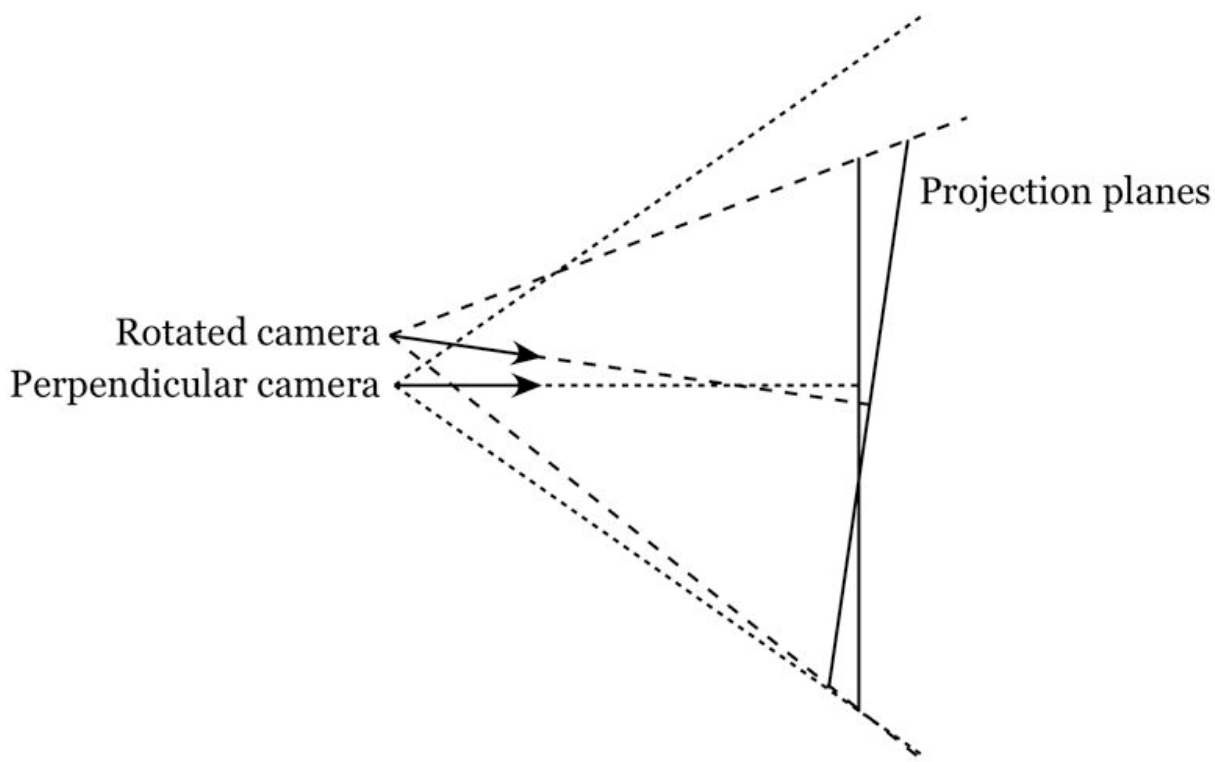

Figure 3. A rotated camera and symmetric frustum do not give the same projected image as the correct asymmetric frustum where the view direction is perpendicular to the projection plane. While the rotated camera may give improved pixel efficiency it does not result in a correct stereo pair on a vertical display screen.

In addition, the dimensions of the viewing screen and the field of view that can be captured by the cameras require a minimum distance between the eventual viewing screen and the observer, for accurate viewing at the fixed position of the force plate within the laboratory. This distance has to be considered in light of the limited laboratory space for equipment placement, as there is a fixed range that certain equipment that make up the stereoscopic projection system have to be placed in relation to one another (e.g. distance of screen to force plate and throw distance of projectors to screen), while still leaving enough room for the successful performance of the Ssg tasks. Therefore, rigorous considerations with regards to the experimental setup need to be made prior to actual filming, as changes to one 
component of the setup will require additional filming. To maximize capture volume and hence reduce the risk of altering the experimental setup, two wider-angled lenses were fitted to the camera pair during filming.

Due to the filming being conducted outdoors, it was not practical to transport the viewing screen for the purposes of matching the view frustums of the cameras to the eventual observers' eyes in relation to the viewing screen. Instead, a more transportable calibration frame (Figure 4) representative of the screen was built for spatial alignment in post processing. The calibration frame had two crossbars, which were adjusted to match the lower and upper edge of the eventual viewing screen. The known aspect ratio of the screen precludes the need for vertical bars for width alignment. During the actual filming session, the frame was videoed on its own for a short period of time, prior to filming actual content. Similar to having the screen in view, the captured calibration frame provided a reference for locking down zero parallax in post processing. Subsequently, the tacklers are filmed performing their defensive movements approximately $55 \mathrm{~cm}$ behind the location of the bar, so that their feet are still in view and no bodily information is lost from the eventual viewer's perspective, for the actual reading of their movements to perform Ssg during the laboratory tests.

A clapper board was also used to mark a sharp event prior to filming actual content for temporal alignment of the left and right capture streams in post-processing. This is due to the lack of genlocking functions, which generally is not supported in small commodity cameras. Using this time alignment method, filming was continuous despite the need for different clips of the various scenarios, as the time and spatial alignment process only needed to be done once instead of multiple times for each stoppage. This saves a significant amount of time in post processing.

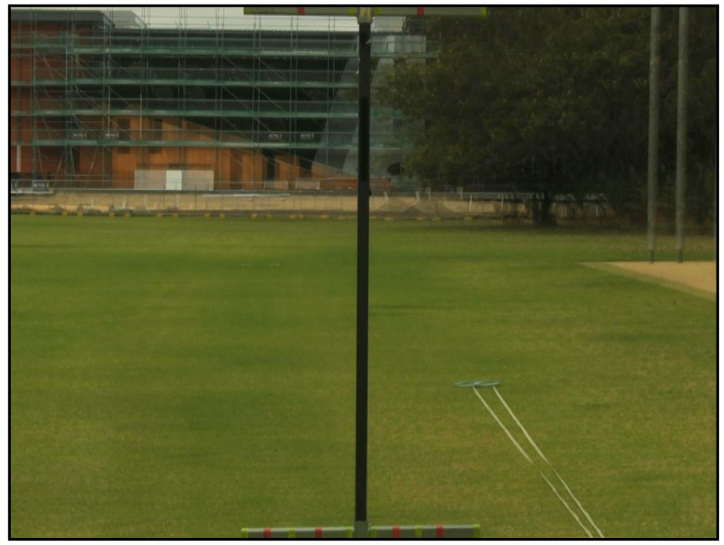

Left and right eye images superimposed

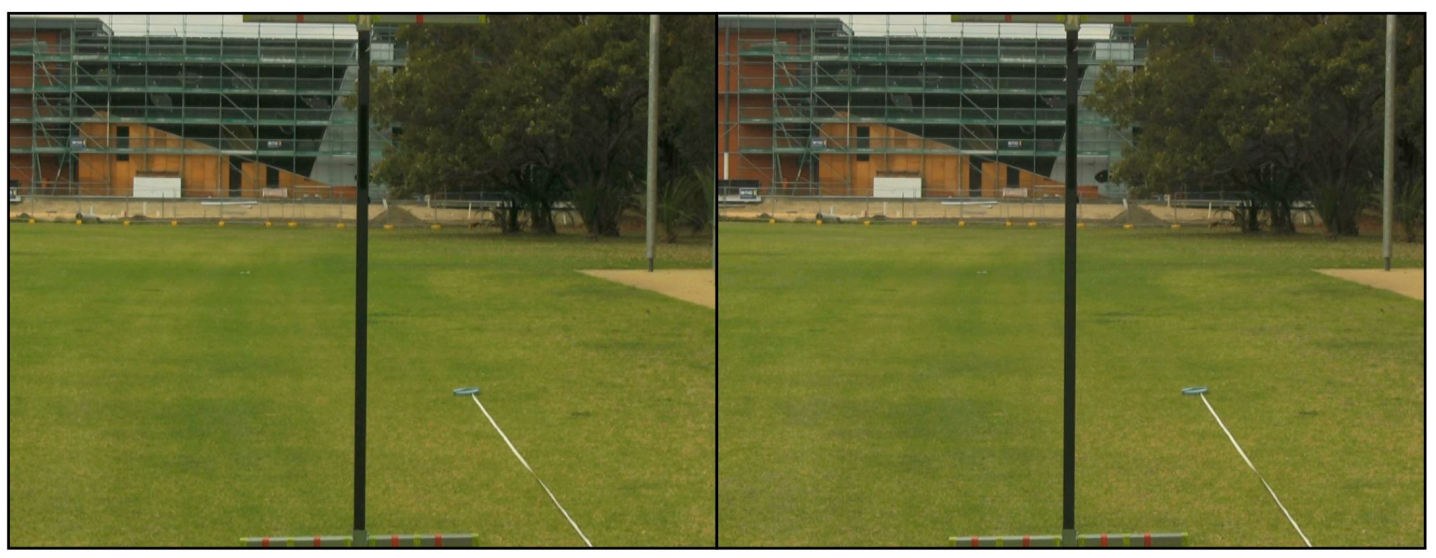

Left and right eye images side-by-side

Figure 4. Left and right eye views after post processing. Top image shows the two images overlaid illustrating perfect zero parallax at the depth of the calibration bar and positive parallax for distant objects. 


\subsection{Stereoscopic playback system}

The presentation hardware used in this project is a standard rear projection, linear polaroid filter based, stereoscopic projection system. Two projectors are used to display footage which are played back as standard movies of double the width or height, For the purposes of this study, double width movie frames were created, with the left eye image captured with the left camera on one half, and the right eye image captured with the right camera on the other half.

To achieve this, when the footage from both streams has been time and space aligned, each stream is exported separately and saved as separate Quicktime movies. Keeping the two streams separate and saving them as master copies allows alterations to be more easily made for alternative project solutions. These are subsequently combined into a single double width movie for playback in Quicktime Pro. The movie files are then "dropped" into custom software written using Quartz Composer to perform the necessary actions for carrying out the evasive Ssg tasks.

\section{CONCLUSIONS AND GENERAL DISCUSSION}

The production of accurate stereoscopic content is often the result of a rigorous process of trial and error [14]. This paper, we believe, not only lays the foundation for further development and use of stereoscopic displays in the sport science domain, but also saves researchers a significant amount of time in the trial and error process, as the key geometric considerations and accompanying steps to achieve these requirements have been presented.

Building on this firm theoretical understanding of stereoscopic filming, this paper presents a cost effective method of producing highly realistic stereoscopic tackle scenarios, for laboratory based investigations of evasive Ssg and associated ACL injury risk. Many sport scientists believe that as technology advances, virtual reality (VR) and simulation systems (i.e. stereoscopic displays) are the future of visual displays used in laboratory based investigations involving perception, including perception resulting in a movement response. Fully immersive VR systems have been used in the context of flight training [22] as well as injury rehabilitation [6]. However, many of these systems either present visual displays that are too simplistic and lack realism (i.e. immersive entertainment/gaming), or are too expensive and cumbersome (i.e. flight simulators), deterring many researchers from actually adopting their use. Moreover, the current study required a spatially and temporally precise life-sized human running towards the viewer and despite their sophistication, VR systems at present cannot replicate the visual texture of an actual human being.

The choice of developing stereoscopic video content using the methods proposed in this paper and presenting it as visual stimuli overcomes the abovementioned limitations. Instead of purchasing expensive equipment to facilitate the filming process, we used existing commodity cameras to perform this highly specific filming process. The development of the metal arm for mounting one of the cameras upside down to achieve a camera separation similar to an average person's inter-ocular distance of $6.5 \mathrm{~cm}$, and the accompanying post processing methodologies presented to achieve the desired end product, are novel yet simple to execute. Other research groups can adopt the methods suggested in this paper to more readily implement the use of stereoscopic projections as a more realistic form of visual display, for investigating laboratory based evasive Ssg and ACL injury risk. It is noteworthy that the stereoscopic system presented in this paper can be adapted for use in any study involving perceptual skill.

Another simple yet significant contribution of this paper is the development of a scene calibration bar for matching the camera frustums to the viewer frustums when filming outdoors, for the creation of the tackle scenarios which possess a true sense of depth and scale to the viewer. The inexpensive and highly transportable scene calibration bar can easily be adjusted to replicate the dimensions of the screen used, thus taking the place of the screen during filming out in the field to lock down zero parallax.

The use of visual displays in laboratory based investigations of perceptual skills and performance efficacy has mainly been limited to the discrimination between experts and novices in various sports. As technology advances, the visual stimuli used have progressed from using simple light based stimuli to two-dimensional life sized video projections, which have yielded interesting results. For example, in a study investigating soccer expertise, it was found that the presentation of cues that reflected more of the game environment, allowed experts to utilise more pertinent selection and more accurate interpretations of the presented visual cues compared with the novices (perceptual component) [11]. This resulted in a more rapid selection of an appropriate response (decision component) thereby allowing experts to execute the required task more smoothly and efficiently (motor component). 
As such, there is a constant drive to improve the ecological validity of visual displays used in laboratory based investigations involving perceptual skill, in a bid to replicate as closely as possible, the actual game environment in the laboratory. Considering that movement efficacy is also related to injury, visual displays used in assessing evasive Ssg and ACL injury risk need to progress from the use of simple light based stimuli to more realistic displays such as stereoscopic displays. .

In recent times, the use of stereoscopic displays has been increasingly used to facilitate procedure in domains such as surgery [24] and education [13]. However, its use in the area of sport science has been limited. Considering that attentional focus has been suggested to be influenced by depth [2], and that three-dimensional stereoscopic images elicit a greater sense of awareness/presence in viewers [8], the use of stereoscopic displays in the research of perceptual skill and movement responses could be the next progression from the use of two-dimensional video projections and light based stimuli.

In summary, we believe that these cost-effective methods presented here allow more research groups studying evasive Ssg and associated ACL injury risk, as well as areas involving perceptual skills resulting in a movement response, to introduce a more realistic form of visual stimuli into laboratories.

\section{LIMITATIONS}

When viewing stereoscopic content, careful matching of the recording geometry and viewing geometry will only create an accurate sense of scale and depth when viewed from a single intended position. All other viewing positions result in some sort of distortion (Figure 5). As one moves towards or away from the selected "correct" position, a depth error occurs in the form of stretching or compression and a scale error occurs due to field of view changes. Movements in all directions from the "correct" viewing position introduce error due to the shearing of space.

Several solutions exist to compensate for this limitation, such as head tracking in real time computer generated stereoscopic pairs. Feedback from head tracking ensures that the correct frustums are always created regardless of viewing points. Unfortunately, a head-tracked stereoscopic display system requires generating images from several arbitrary viewpoints, which limits its use mainly to computer generated content [17]. With filmed material, this option is not available.

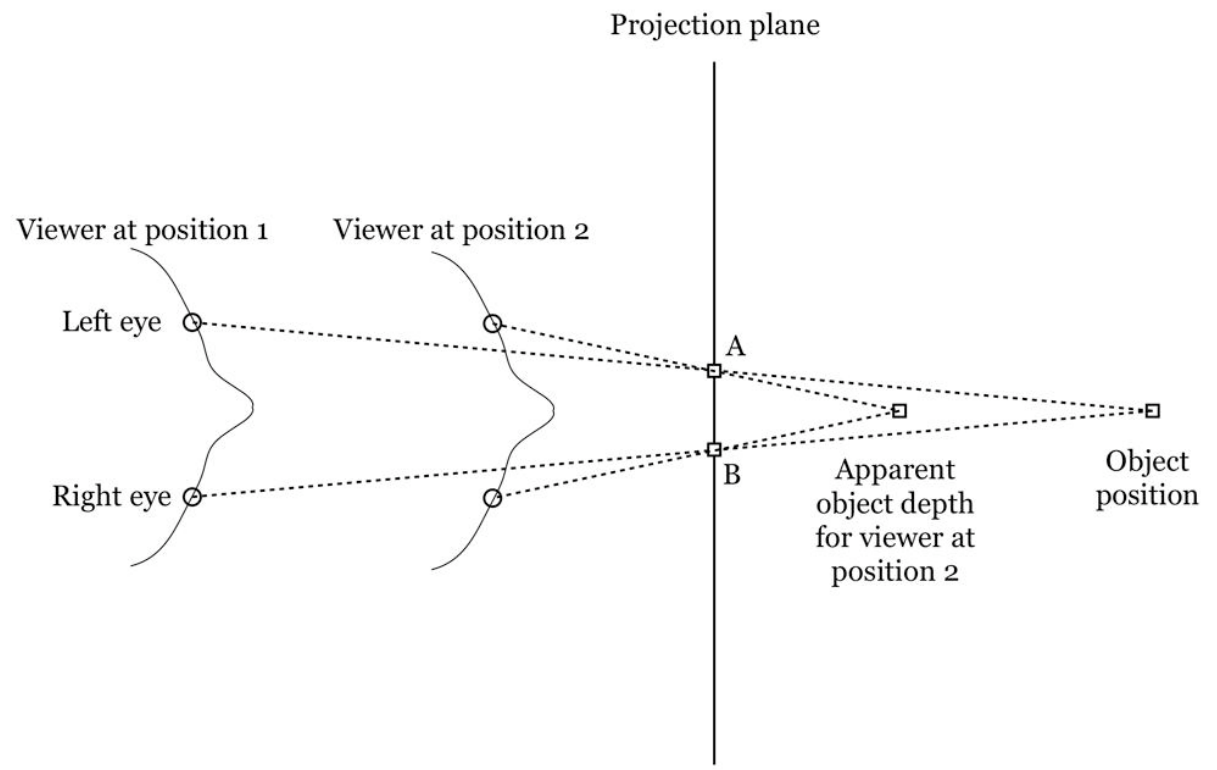

Figure 5. Demonstration of the compression of depth. An object at the position indicated on the right presents itself at position $\mathrm{A}$ and $\mathrm{B}$ on the left and right eye projection plane. If the viewer moves to position 2 the virtual objects appears at a different apparent depth. As the viewer moves closer or further from the position for which the image is captured, the depth contracts or expands. 


\section{FUTURE WORK}

Currently, the stereoscopic tackle scenarios possess an accurate sense of scale and depth when viewed at the selected "correct" position, which is the position of the force plate in the laboratory, where the reactive side-step is executed. We will endeavour to correct for distortions when the viewer moves away from the "correct" position, such that the camera's recording geometry is matched to the eventual viewing geometry at every point between the convergence of the viewer and the tackler/s. If proven successful, the stereoscopic scenarios should exhibit a true sense of depth and scale similar to what the athlete would see in real life, at every point during the run up while reading the movements of the tackler/s and finally performing the evasive side-step in the laboratory.

In addition, the stereoscopic system will be assimilated with other motion analyses tools to assess if differences exist in the perception and movement response couplings, when the stereoscopic tackle scenarios are presented as visual stimuli instead of the traditional light based stimuli, to assess evasive Ssg in the laboratory. We believe that the stereoscopic tackle scenarios will elicit knee loadings as well as muscle stabilization strategies that are more similar to what happens in actual game environments, when the athlete has to prepare and perform an evasive side-step. To date, no study has linked perceptual skills to ACL injury risk. Neither has a stereoscopic display been used as a more realistic form of visual display for the study of perception and movement response couplings such as evasive Ssg. We believe that the development of this tool will allow subsequent studies to optimise the ecological validity of laboratory based investigations of reactive Ssg and ACL injury risk, as well as any other studies involving visual perception.

\section{REFERENCES}

[1] Arendt, E.A., Agel, J. and Dick, R., "Anterior cruciate ligament injury patterns among collegiate men and women," Journal of Athletic Training 34, 88-92 (1999).

[2] Atchley, P. and Kramer, A.F., "Spatial cuing in a stereoscopic display: Evidence for a "depth-aware" attentional focus," Psychonomic Bulletin \& Review 4(4), 524-529 (1997).

[3] Besier, T.F., Lloyd, D.G., Cochrane, J.L. and Ackland, T.R., "External loading of the knee joint during running and cutting manoeuvres," Medicine and Science in Sports and Exercise 33, 1168-1175 (2001a).

[4] Besier, T.F., Lloyd, D.G., Cochrane, J.L. and Ackland, T.R., "Anticipatory effects on knee joint loading during running and cutting manoeuvres," Medicine and Science in Sports and Exercise 33, 1176-1181 (2001b).

[5] Besier, T.F., Lloyd, D.G. and Ackland, T.R., "Muscle activation strategies at the knee during running and cutting manoeuvres," Medicine and Science in Sports and Exercise 35, 119-127 (2003).

[6] Burdea, G., Popescu, V., Hentz, V. and Colbert, K., "Virtual Reality-Based Orthopedic Telerehabilitation," IEEE Transactions on Rehabilitation Engineering, 430-432 (2000).

[7] Cochrane, J.L., Lloyd, D.G., Buttfield, A., Seward, H. and McGivern, J., "Characteristic of anterior cruciate ligament injuries in Australian Football," Journal of Science and Medicine in Sport 10, 96-104 (2007).

[8] Freeman, J., Avons, S.E., Pearson, D. and Ijsselsteijin, W.A., "Effects of sensory information and prior experience on direct subjective ratings of presence," Presence: Teleoperators and Virtual Environments (8), 1-13 (1999).

[9] Gillquist, J. and Messner, K., "Anterior cruciate ligament reconstruction and the long term incidence of gonarthrosis," Sports Medicine 27, 143-156 (1999).

[10] Gray, R. and Regan, D., "Accuracy of estimating time to collision using binocular and monocular information," Vision Research 38, 4, 499-512 (1998).

[11] Helsen, W.F. and Starkes, J.L., "A multidimensional approach to skilled perception and performance in sport," Applied Cognitive Psychology 13, 1-27 (1999).

[12] Hewett, T.E., Lindenfeld, T.N., Ricobene, J.V. and Noyes, F.R., "The effect of neuromuscular training on the incidence of knee injury in female athletes: a prospective study," American Journal of Sports Medicine 27, 699706 (1999).

[13] Ilgner, J., Kawai, T., Shibata, T., Yamazoe, T. and Westhofen, M., "Evaluation of stereoscopic medical video content on an autostereoscopic display for undergraduate medical education," Proc. SPIE 6055, (2006)

[14] Jones, G., Lee, D., Holliman, N. and Ezra, D., "Controlling Perceived Depth in Stereoscopic Images," Proc. SPIE 4297A, (2001).

[15] Kandel, E.R., Schwartz, J.H. and Jessell, T.M., [Principles of Neural Science], McGraw-Hill, New York, (2000). 
[16] Lloyd, D.G., "Rationale for training programmes to reduce ACL injuries in Australian Football," Journal of Orthopaedic and Sports Physical Therapy, 645-654 (2001).

[17] Mclean, S.G., Lipfert, S.W. and Van Den Bogert, A.J., "Effect of gender and defensive opponent on the biomechanics of sidestep cutting," Medicine and Science in Sports and Exercise 36, 1008-1016 (2004).

[18] McMillan, L. and Bishop, G., "Head-tracked stereoscopic display using image warping," Proc. SPIE 2409, 21-30 (1995).

[19] Mihata, L.C.S., Beautler, A.I. and Boden, B.P., "Comparing the incidence of anterior cruciate ligament injury in collegiate lacrosse, soccer, and basketball players: Implications for anterior cruciate ligament mechanism and prevention," American Journal of Sports Medicine 34, 899-904 (2006).

[20] Orchard, J. and Seward, H., “AFL injury report 2007," Melbourne: AFLMOA (2008).

[21] Olsen, O-E., Myklebust, G., Engebretsen, L. and Bahr, R., "Injury mechanisms for anterior cruciate ligament injuries in team handball: A systematic video analysis," American Journal of Sports Medicine 32, 1002-1012 (2004).

[22] Schachter, B.J., "Computer Image Generation for Flight Simulation," IEEE Computer Graphics and Applications 1(4), (1981).

[23] Wallach, H., Moore, M.E. and Davidson, L., "Modification of Stereoscopic Depth-Perception," The American Journal of Sport Psychology 76(2), 191-204 (1963).

[24] Yaron, A., Bar-Zohar, M. and Horesh, N., "Miniature stereoscopic video system provides real-time 3D registration and image fusion for minimally invasive surgery," Proc. SPIE 6055, (2006). 